\title{
COMPLEX INTERPOLATION OF A BANACH SPACE WITH ITS DUAL
}

\author{
FRÉDÉRIQUE WATBLED
}

\begin{abstract}
Let $X$ be a Banach space compatible with its antidual $\overline{X^{\star}}$, where $\overline{X^{\star}}$ stands for the vector space $X^{\star}$ where the multiplication by a scalar is replaced by the multiplication $\lambda \odot x^{\star}=\bar{\lambda} x^{\star}$. Let $H$ be a Hilbert space intermediate between $X$ and $\overline{X^{\star}}$ with a scalar product compatible with the duality $\left(X, X^{\star}\right)$, and such that $X \cap \overline{X^{\star}}$ is dense in $H$. Let $F$ denote the closure of $X \cap \overline{X^{\star}}$ in $\overline{X^{\star}}$ and suppose $X \cap \overline{X^{\star}}$ is dense in $X$. Let $K$ denote the natural map which sends $H$ into the dual of $X \cap F$ and for every Banach space $A$ which contains $X \cap F$ densely let $A^{\prime}$ be the realization of the dual space of $A$ inside the dual of $X \cap F$. We show that if $\left|\left\langle K^{-1} a, K^{-1} b\right\rangle_{H}\right| \leq\|a\|_{X^{\prime}}\|b\|_{F^{\prime}}$ whenever $a$ and $b$ are both in $X^{\prime} \cap F^{\prime}$ then $\left(X, \overline{X^{\star}}\right)_{\frac{1}{2}}=H$ with equality of norms. In particular this equality holds true if $X$ embeds in $H$ or $H$ embeds densely in $X$. As other particular cases we mention spaces $X$ with a 1 -unconditional basis and Köthe function spaces on $\Omega$ intermediate between $L^{1}(\Omega)$ and $L^{\infty}(\Omega)$.
\end{abstract}

\section{Introduction}

We first recall the basic definitions of the Calderón complex interpolation method, which can be found in [4], [3] (Cf. also [7], [10]). We say that two Banach spaces $A_{0}, A_{1}$ are compatible if there exists a Hausdorff topological vector space $\mathscr{U}$ and continuous linear injections $i_{0}$ of $A_{0}$ into $\mathscr{U}$ and $i_{1}$ of $A_{1}$ into $\mathscr{U}$ which allow us to identify $A_{0}$ and $A_{1}$ with vector subspaces of $\mathscr{U}$. We can then give sense to the intersection and the sum of $A_{0}$ and $A_{1}$ which become Banach spaces equipped with the following norms:

$$
\begin{gathered}
\|a\|_{A_{0} \cap A_{1}}=\max \left(\|a\|_{A_{0}},\|a\|_{A_{1}}\right), \\
\|a\|_{A_{0}+A_{1}}=\inf \left(\left\|a_{0}\right\|_{A_{0}}+\left\|a_{1}\right\|_{A_{1}}, a=a_{0}+a_{1}, a_{j} \in A_{j}\right) .
\end{gathered}
$$

If $A_{0} \cap A_{1}$ is dense in $A_{0}$ and $A_{1}$ then the dual of $A_{0} \cap A_{1}$ can be identified with $A_{0}^{\star}+A_{1}^{\star}$ and the dual of $A_{0}+A_{1}$ can be identified with $A_{0}^{\star} \cap A_{1}^{\star}$, which provides a scheme where $A_{0}^{\star}$ and $A_{1}^{\star}$ are compatible. We say that a space $A$ is intermediate between $A_{0}$ and $A_{1}$ if $A_{0} \cap A_{1} \subset A \subset A_{0}+A_{1}$ with continuous inclusions. Let $S=\{z \in \mathrm{C}, 0 \leq \Re z \leq 1\}, \quad S_{0}=\{z \in \mathrm{C}, 0<\Re z<1\}$. If $\left(A_{0}, A_{1}\right)$ is a compatible couple of complex Banach spaces, $\mathscr{F}\left(A_{0}, A_{1}\right)$ de-

Received January 13, 1998. 
notes the family of functions defined on $S$, continuous and bounded with values in $A_{0}+A_{1}$, holomorphic on $S_{0}$, such that the functions $t \mapsto f(j+i t)$, $j=0,1$, are continuous functions from $\mathrm{R}$ to $A_{j}$ which tend to 0 as $|t| \rightarrow+\infty$. The space $\mathscr{F}\left(A_{0}, A_{1}\right)$ is a Banach space under the norm

$$
\|f\|_{\mathscr{F}\left(A_{0}, A_{1}\right)}=\max _{j=0,1} \sup _{t \in \mathrm{R}}\|f(j+i t)\|_{A_{j}},
$$

and the complex interpolation spaces are defined for $\theta \in[0,1]$ by

$$
\left(A_{0}, A_{1}\right)_{\theta}=\left\{f(\theta), f \in \mathscr{F}\left(A_{0}, A_{1}\right)\right\},
$$

which are Banach spaces under the norm

$$
\|a\|_{[\theta]}=\inf \left\{\|f\|_{\mathscr{F}\left(A_{0}, A_{1}\right)}, f \in \mathscr{F}\left(A_{0}, A_{1}\right), f(\theta)=a\right\} .
$$

Let us denote by $\mathscr{F}_{0}\left(A_{0}, A_{1}\right)$ the family of functions in $\mathscr{F}\left(A_{0}, A_{1}\right)$ of the form $F(z)=\sum_{k=1}^{n} F_{k}(z) a_{k}$, with $F_{k}$ in $\mathscr{F}(\mathrm{C}, \mathrm{C})$ and $a_{k}$ in $A_{0} \cap A_{1}$. Calderón showed that $\mathscr{F}_{0}\left(A_{0}, A_{1}\right)$ is dense in $\mathscr{F}\left(A_{0}, A_{1}\right)$, which implies of course that $A_{0} \cap A_{1}$ is dense in every $\left(A_{0}, A_{1}\right)_{\theta}$. Moreover, if $X^{0}$ denotes the closure of $A_{0} \cap A_{1}$ in $X$ then

$$
\left(A_{0}, A_{1}\right)_{\theta}=\left(A_{0}^{0}, A_{1}\right)_{\theta}=\left(A_{0}, A_{1}^{0}\right)_{\theta}=\left(A_{0}^{0}, A_{1}^{0}\right)_{\theta}
$$

with equality of norms. We shall also need the second Calderón interpolation method: let us denote by $\mathscr{G}\left(A_{0}, A_{1}\right)$ the family of functions $g$ continuous on $S$ with values in $A_{0}+A_{1}$, holomorphic on $S_{0}$, such that $\|g(z)\|_{A_{0}+A_{1}} \leq$ $c(1+|z|), g\left(j+i t_{1}\right)-g\left(j+i t_{2}\right) \in A_{j}$ for $t_{1}, t_{2} \in \mathrm{R}, j=0,1$, and

$$
\|g\|_{\mathscr{G}\left(A_{0}, A_{1}\right)}=\max _{j=0,1} \sup _{t_{1}, t_{2} \in \mathrm{R}, t_{1} \neq t_{2}}\left\|\frac{g\left(j+i t_{1}\right)-g\left(j+i t_{2}\right)}{t_{1}-t_{2}}\right\|_{A_{j}}<\infty .
$$

The space $\mathscr{G}\left(A_{0}, A_{1}\right)$ reduced modulo the constant functions and equipped with the norm above is a Banach space and the second complex interpolation spaces are defined by

$$
\left(A_{0}, A_{1}\right)^{\theta}=\left\{g^{\prime}(\theta), g \in \mathscr{G}\right\},
$$

which are Banach spaces under the norm

$$
\|a\|^{[\theta]}=\inf \left\{\|g\|_{\mathscr{G}\left(A_{0}, A_{1}\right)}, g \in \mathscr{G}, g^{\prime}(\theta)=a\right\} .
$$

The second method of interpolation is needed to identify the dual of an interpolation space: indeed the duality theorem asserts that if $A_{0} \cap A_{1}$ is dense in both $A_{0}$ and $A_{1}$ then $\left(A_{0}, A_{1}\right)_{\theta}^{\star}=\left(A_{0}^{\star}, A_{1}^{\star}\right)^{\theta}$ for every $\left.\theta \in\right] 0,1$ [ with equality of norms. Calderón showed the inclusion $\left(A_{0}, A_{1}\right)_{\theta} \subset\left(A_{0}, A_{1}\right)^{\theta}$ and Bergh ([1]) proved that $\|a\|_{[\theta]}=\|a\|^{[\theta]}$ for every $a \in\left(A_{0}, A_{1}\right)_{\theta}$. It is well known that 
equality holds if one of the spaces $A_{0}, A_{1}$ is reflexive, but there is still no satisfactory characterization of spaces for which equality holds (see [2] for a survey).

Here we investigate another well known fact: the space $\left(L_{p}, L_{q}\right)_{\frac{1}{2}}$ is isometric to $L_{2}$ for every $\left.p \in\right] 1,+\infty\left[\right.$ and $\frac{1}{p}+\frac{1}{q}=1$. More generally, if $X$ is a reflexive Banach space compatible with its antidual $\overline{X^{\star}}$, that is the vector space $X^{\star}$ where the multiplication $\lambda x, \lambda \in \mathrm{C}, x \in X^{\star}$, is replaced by the conjugate multiplication $\bar{\lambda} x$, then $\left(X, \overline{X^{\star}}\right)_{\frac{1}{2}}$ is isometric with a Hilbert space provided $X \cap \overline{X^{\star}}$ is dense in $X$ and in $\overline{X^{\star}}$. Pisier has showned (in [6] with Haagerup with a supplementary hypothesis, and in [9] in full generality) that if there is a continuous injection $v$ of a Hilbert space $H$ into $X$ with dense range, and if we identify $\overline{X^{\star}}$ with the subspace $v v^{\star}\left(\overline{X^{\star}}\right)$ of $X$, then the equality $\left(X, \overline{X^{\star}}\right)_{\frac{1}{2}}=H$ holds again. In my thesis ([12]) I proved this equality in several other cases, in particular when $X$ embeds in $H$ (Cf. also [11]), or when $X$ is a space with a 1-unconditional basis, or when $X$ is a $\sigma$-order continuous rearrangement invariant Köthe function space. I also proved the equality when $X \cap \overline{X^{\star}}$ is dense in $X$ and $\overline{X^{\star}}$ and with a supplementary hypothesis, but a simpler proof was given afterwards independently by Cobos and Schonbek ([5]). The main result of this paper is that equality holds if $X \cap \overline{X^{\star}}$ is dense in $X$ and $\left|\left\langle K^{-1} a, K^{-1} b\right\rangle_{H}\right| \leq\|a\|_{X^{\prime}}\|b\|_{F^{\prime}}$ as soon as $a$ and $b$ are both in $X^{\prime} \cap F^{\prime}$ (Theorem 1), where $F$ stands for the closure of $X \cap \overline{X^{\star}}$ in $\overline{X^{\star}}, X^{\prime}, F^{\prime}$, are the realizations of the duals of $X$ and $F$ inside the dual of $X \cap F$, and $K$ is the natural isometry of $H$ onto $H^{\prime}$. This hypothesis holds in every case mentioned above and also in the case of a general Köthe space $X$ such that $X \cap \overline{X^{\star}}$ is dense in $X$.

\section{Complex interpolation of a Banach space with its dual}

In all this section we shall assume that the Banach space $X$ is compatible with its antidual $\overline{X^{\star}}$, and that there exists a Hilbert space $H$ intermediate between $X$ and $\overline{X^{\star}}$. Thus, as explained above, for some Hausdorff topological vector space $\mathscr{U}$, there exist continuous linear injections $i_{0}: X \rightarrow \mathscr{U}$ and $i_{1}: \overline{X^{\star}} \rightarrow \mathscr{U}$ such that $i_{0}(X) \cap i_{1}\left(\overline{X^{\star}}\right) \subset H \subset i_{0}(X)+i_{1}\left(\overline{X^{\star}}\right) \subset \mathscr{U}$. But in fact it is possible to simplify this notation and our presentation. We first observe that, without loss of generality, we can suppose, by redefining $X$, that $X \subset \mathscr{U}$ and that $i_{0}$ is the identity operator. The next step is to also make $i_{1}$ become the identity operator, by suitably adjusting the bilinear or sesquilinear mapping which is used to define the action of linear functionals on $X$. More specifically, let $Y=i_{1}\left(\overline{X^{\star}}\right)$ and norm $Y$ so that $i_{1}$ is an isometry. Define a map 


$$
\begin{aligned}
\psi: X \times Y & \rightarrow \mathrm{C} \\
(x, y) & \mapsto i_{1}^{-1}(y)(x),
\end{aligned}
$$

i.e. $\psi(x, y)$ is the value of the functional $i_{1}^{-1}(y) \in \overline{X^{\star}}$ when applied to the element $x \in X$. Since $i_{1}: \overline{X^{\star}} \rightarrow Y$ is linear, $\psi$ is sesquilinear with $\psi(\lambda x, y)=$ $\lambda \psi(x, y)=\psi(x, \bar{\lambda} y)$. Thus, if we decide to define the action of all bounded linear functionals on $X$ in terms of $\psi$, we can then in fact write $Y=\overline{X^{\star}}$, so that $X$ and $\overline{X^{\star}}$ are subspaces of $\mathscr{U}$.

Now we can define what we mean by a scalar product compatible with the duality:

Definition. Let $X$ be a Banach space compatible with its antidual $\overline{X^{\star}}$ and $H$ be a Hilbert space intermediate between $X$ and $\overline{X^{\star}}$. We say that the scalar product of $H$ is compatible with the duality $\left(X, X^{\star}\right)$ if for every $h \in H$ such that $h=x+x^{\star}$ with $x \in X$ and $x^{\star} \in \overline{X^{\star}}$, we have

$$
\langle h, a\rangle_{H}=\psi(x, a)+\overline{\psi\left(a, x^{\star}\right)} \text { for every } a \in X \cap \overline{X^{\star}} .
$$

REMARK. The existence of an intermediate Hilbert space with a scalar product compatible with the duality $\left(X, \overline{X^{\star}}\right)$ implies that $\left(X \cap \overline{X^{\star}}, \psi\right)$ is a prehilbertian space since

$$
\langle h, a\rangle_{H}=\psi(h, a)=\overline{\psi(a, h)} \text { for every } a, h \in X \cap \overline{X^{\star}} .
$$

Conversely if $\left(X \cap \overline{X^{\star}}, \psi\right)$ is a prehilbertian space then its completion $H$ is a Hilbert space, but there is no reason why this $H$ should continuously embed into $X+\overline{X^{\star}}$.

From now on we assume that the scalar product of our intermediate Hilbert space $H$ is compatible with the duality $\left(X, X^{\star}\right)$. We assume also without loss of generality that $X \cap \overline{X^{\star}}$ is dense in $H$. Then we can easily obtain the following:

Lemma. In the above setting, we have $\left(X, \overline{X^{\star}}\right)_{\frac{1}{2}} \subset H$ with norm less than or equal to one.

Proof. As Pisier in [9], we shall use the bilinear interpolation theorem of Calderón. Let us first explain how to adapt it to the case of sesquilinear mappings. For any topological space $B$, let $\bar{B}$ denote the topological vector space which is $B$ equipped with same topology (or norm) and with the operation $\lambda \odot b=\bar{\lambda} b$ for multiplication by scalars. Then $\left(A_{0}, A_{1}\right)$ is a couple of Banach spaces contained in $\mathscr{U}$ if and only if $\left(\overline{A_{0}}, \overline{A_{1}}\right)$ is such a couple contained in $\overline{\mathscr{U}}$. Next, consider an arbitrary element $F \in \mathscr{F}_{0}\left(A_{0}, A_{1}\right)$, i.e. $F(z)=$ $\sum_{k=1} F_{k}(z) a_{k}$ where $F_{k} \in \mathscr{F}(\mathrm{C}, \mathrm{C})$ and $a_{k} \in A_{0} \cap A_{1}$. Define $G: S \mapsto A_{0}+A_{1}$ by setting $G(z)=\sum_{k=1}^{n} \overline{F_{k}(\bar{z})} \odot a_{k}$. Then clearly $G \in \mathscr{F}_{0}\left(\overline{A_{0}}, \overline{A_{1}}\right)$ and 
$G(\theta)=F(\theta)$. Furthermore $\|G\|_{\mathscr{F}_{0}\left(\overline{A_{0}}, \overline{A_{1}}\right)}=\|F\|_{\mathscr{F}_{0}\left(A_{0}, A_{1}\right)}$. By considering all such $F$ and $G$ it is easy to show that

$$
\overline{\left(A_{0}, A_{1}\right)_{\theta}}=\left(\overline{A_{0}}, \overline{A_{1}}\right)_{\theta}
$$

with equality of norms. Hence one can deduce an interpolation theorem for sesquilinear mappings from Calderon's theorem and the fact that for any Banach spaces $A$ and $B$ a map $\phi: A \times B \rightarrow \mathrm{C}$ is sesquilinear if and only if it is bilinear as a map from $A \times \bar{B}$ to $\mathrm{C}$.

Now the sesquilinear form $\varphi$ defined on $X \cap \overline{X^{\star}} \times \overline{X^{\star}} \cap X$ by $\varphi(a, b)=$ $\langle a, b\rangle_{H}$ is bounded with norm less than or equal to one both on $X \times \overline{X^{\star}}$ and on $\overline{X^{\star}} \times X$ so that it extends by the bilinear interpolation theorem to a sesquilinear form of norm less than or equal to one on $\left(X, \overline{X^{\star}}\right)_{\frac{1}{2}} \times\left(\overline{X^{\star}}, X\right)_{\frac{1}{2}}=$ $\left(\left(X, \overline{X^{\star}}\right)_{\frac{1}{2}}\right)^{2}$. In particular we have for every $x$ in $X \cap \overline{X^{\star}}, \varphi(x, x)=$ $\|x\|_{H}^{2} \leq\|x\|_{\left(X, \overline{X^{\star}}\right)_{\frac{1}{2}}}^{2}$, hence $\|x\|_{H} \leq\|x\|_{\left(X, \overline{X^{\star}}\right)_{\frac{1}{2}}}$. As $X \cap \overline{X^{\star}}$ is dense in $\left(X, \overline{X^{\star}}\right)_{\frac{1}{2}}$ and as $H$ and $\left(X^{2}, \overline{X^{\star}}\right)_{\frac{1}{2}}$ are both continuously imbedded in $X+\overline{X^{\star}}$ we deduce that $\left(X, \overline{X^{\star}}\right)_{\frac{1}{2}}$ is included in $H$ with $\|x\|_{H} \leq\|x\|_{\left(X, \overline{X^{\star}}\right)_{\frac{1}{2}}}$ for every $x$ in $\left(X, \overline{X^{\star}}\right)_{\frac{1}{2}}$.

In the sequel we shall make the supplementary assumption that $X \cap \overline{X^{\star}}$ is dense in $X$, and we shall let $F$ denote the closure of $X \cap \overline{X^{\star}}$ in $\overline{X^{\star}}$. Now the couple we are really interested in is the couple $(X, F)$, since we have $X \cap \overline{X^{\star}}=X \cap F$, and $\left(X, \overline{X^{\star}}\right)_{\frac{1}{2}}=(X, F)_{\frac{1}{2}}$. The space $H$ is continuously included in $X+\overline{X^{\star}}, X \cap F$ is dense in $H$, and $X+F$ is a closed subspace of $X+\overline{X^{\star}}$ (because the norm of $X+F$ is equal to the norm of $X+\overline{X^{\star}}$ : indeed if $x+f=y+y^{\star}$ with $x, y \in X, f \in F, y^{\star} \in \overline{X^{\star}}$ then necessarily $y^{\star} \in F$ since $\left.x-y=y^{\star}-f \in X \cap \overline{X^{\star}}=X \cap F\right)$, therefore we obtain that $H \subset X+F$ (continuous inclusion). As $X \cap F$ is dense both in $X$ and in $F$ it is also dense in $X+F$, and $H$ which contains $X \cap F$ is therefore dense in $X+F$. Let $\mathscr{V}$ be the dual space of $X \cap F$ and let us denote the action of $v \in \mathscr{V}$ on $x \in X \cap F$ by $\gamma(x, v)$, so that

$$
\gamma: X \cap F \times \mathscr{V} \rightarrow \mathrm{C}
$$

is a bilinear form. For each normed space $A$ which contains $X \cap F$ densely, let $A^{\prime}$ denote the subspace of $\mathscr{V}$ consisting of those elements $v$ for which the norm

$$
\|v\|_{A^{\prime}}=\sup \left\{|\gamma(x, v)|: x \in X \cap F,\|x\|_{A} \leq 1\right\}
$$

is finite. Then $A^{\prime}$ is a realization of the dual space of $A$. In particular we will consider and use the space $A^{\prime}$ when $A$ is any of the spaces $X, F, X+F$ and $H$. The two spaces $X^{\prime}$ and $F^{\prime}$ form a compatible couple with $\mathscr{V}$ as their containing space, and we have $(X+F)^{\prime}=X^{\prime} \cap F^{\prime},(X \cap F)^{\prime}=\mathscr{V}=X^{\prime}+F^{\prime}$, 
$\left((X, F)_{\frac{1}{2}}\right)^{\prime}=\left(X^{\prime}, F^{\prime}\right)^{\frac{1}{2}}$. Also the continuous inclusion $H \subset X+F$ implies the continuous inclusion $X^{\prime} \cap F^{\prime} \subset H^{\prime}$. Now since $X \cap F$ is continuously included in $H$, each $h \in H$ defines an element $K h \in \mathscr{V}$ such that

$$
\gamma(x, K h)=\langle x, h\rangle_{H} \text { for all } x \in X \cap F .
$$

This defines a one to one operator $K$ which is an antilinear isometry of $H$ onto $H^{\prime}$. We are ready for theorem 1 :

THeorem 1. Let $X$ be a Banach space compatible with $\overline{X^{\star}}$ such that $X \cap \overline{X^{\star}}$ is dense in $X$, and let $F$ be the closure of $X \cap \overline{X^{\star}}$ in $\overline{X^{\star}}$. Let $H$ be an intermediate Hilbert space between $X$ and $\overline{X^{\star}}$ with a scalar product compatible with the duality $\left(X, X^{\star}\right)$ and $X \cap \overline{X^{\star}}$ dense in $H$. If

$$
\left|\left\langle K^{-1} a, K^{-1} b\right\rangle_{H}\right| \leq\|a\|_{X^{\prime}}\|b\|_{F^{\prime}} \text { for all } a, b \in X^{\prime} \cap F^{\prime}
$$

then $\left(X, \overline{X^{\star}}\right)_{\frac{1}{2}}=H$ with equality of norms.

Proof. The sesquilinear form $\varphi$ defined on $X^{\prime} \cap F^{\prime} \times F^{\prime} \cap X^{\prime}$ by $\varphi(a, b)=$ $\left\langle K^{-1} a, K^{-1} b\right\rangle_{H}$ is bounded with norm less than or equal to one both on $X^{\prime} \times F^{\prime}$ and on $F^{\prime} \times X^{\prime}$ by hypothesis so it extends by the bilinear interpolation theorem to a sesquilinear form of norm less than or equal to one on $\left(X^{\prime}, F^{\prime}\right)_{\frac{1}{2}} \times\left(F^{\prime}, X^{\prime}\right)_{\frac{1}{2}}=\left(\left(X^{\prime}, F^{\prime}\right)_{\frac{1}{2}}\right)^{2}$. Using the same arguments as in the proof of the inclusion

$$
(X, F)_{\frac{1}{2}} \subset H \text { with norm } \leq 1
$$

we deduce the inclusion

$$
\left(X^{\prime}, F^{\prime}\right)_{\frac{1}{2}} \subset H^{\prime} \text { with norm } \leq 1 .
$$

On the other hand by dualizing the inclusion $(X, F)_{\frac{1}{2}} \subset H$ we obtain $H^{\prime} \subset\left(X^{\prime}, F^{\prime}\right)^{\frac{1}{2}}$ with norm less than or equal to one. As $\left(X^{\prime}, F^{\prime}\right)_{\frac{1}{2}}$ is a subspace of $\left(X^{\prime}, F^{\prime}\right)^{\frac{1}{2}}$ with the same norm, this implies the equality

$$
\|x\|_{H^{\prime}}=\|x\|_{\left(X^{\prime}, F^{\prime}\right)_{\frac{1}{2}}} \text { for every } x \in\left(X^{\prime}, F^{\prime}\right)_{\frac{1}{2}} \text {. }
$$

Now $\left(X^{\prime}, F^{\prime}\right)_{\frac{1}{2}}$ is reflexive hence equal to $\left(X^{\prime}, F^{\prime}\right)^{\frac{1}{2}}$ thanks to the proposition below, so that eventually $\left((X, F)_{\frac{1}{2}}\right)^{\prime}$ is equal to $H^{\prime}$ with equality of norms, and so we obtain $(X, F)_{\frac{1}{2}}=H$.

For the sake of completeness let us state as a proposition the result we used in the previous proof (cf. also [12], Proposition II.1.3):

Proposition. Let $A_{0}, A_{1}$ be two compatible Banach spaces with $A_{0} \cap A_{1}$ dense in $A_{0}$ and $A_{1}$, let $\left.\theta \in\right] 0,1\left[\right.$. If $\left(A_{0}^{\star}, A_{1}^{\star}\right)_{\theta}$ is reflexive then $\left(A_{0}^{\star}, A_{1}^{\star}\right)_{\theta}=$ $\left(A_{0}^{\star}, A_{1}^{\star}\right)^{\theta}$. 
Proof. We know that $\left(A_{0}^{\star}, A_{1}^{\star}\right)_{\theta}$ is a subspace of $\left(A_{0}^{\star}, A_{1}^{\star}\right)^{\theta}$ with the same norm, and we also know ([13], Lemma 2 or [11], [12]) that it is sequentially dense in $\left(A_{0}^{\star}, A_{1}^{\star}\right)^{\theta}$ for the weak star topology $\sigma\left(\left(A_{0}^{\star}, A_{1}^{\star}\right)^{\theta},\left(A_{0}, A_{1}\right)_{\theta}\right)$. Now if $Y$ is a closed reflexive subspace of a dual Banach space $X^{\star}$ which is also sequentially weak star dense in $X^{\star}$ then $Y$ is equal to $X^{\star}$.

Theorem 1 implies the result of Pisier mentioned in the introduction:

Corollary 1. Let $H$ be a Hilbert space, let $v: H \rightarrow X$ be an injection with dense range, and $H_{1}=v(H)$. If we identify $\overline{X^{\star}}$ with the subspace of $X$ defined by

$$
\overline{X^{\star}}=\left\{y \in H_{1},\left|\langle x, y\rangle_{H_{1}}\right| \leq C\|x\|_{X} \forall x \in H_{1}\right\}
$$

then $\left(X, \overline{X^{\star}}\right)_{\frac{1}{2}}=H_{1}$ with equality of norms.

Proof. Here we have $\overline{X^{\star}} \subset H_{1} \subset X$ with continuous inclusions and $H_{1}$ dense in $X, X \cap \overline{X^{\star}}=\overline{X^{\star}}, X+\overline{X^{\star}}=X, F=\overline{X^{\star}}$. The scalar product of $H_{1}$ is compatible with the duality $\left(X, X^{\star}\right)$ by definition of $\overline{X^{\star}}$, and $\overline{X^{\star}}$ is dense in $H_{1}$ because every linear functional $F$ bounded on $H_{1}$ which vanishes on $\overline{X^{\star}}$ is of the form $F(h)=\langle h, k\rangle_{H_{1}}$ with $k \in H_{1}$ hence if $\langle h, k\rangle_{H_{1}}=0$ for every $h \in \overline{X^{\star}}$ then the value of the linear form $h$ on $k \in X$ is zero for every $h \in \overline{X^{\star}}$ and therefore $k=0$, i.e. $F=0$. The space $\overline{X^{\star}}$ is also dense in $X$, and it is easy to check that $K$ is an isometry from $\overline{X^{\star}}$ onto $X^{\prime}$, so that for every $a, b \in X^{\prime} \cap \overline{X^{\star}}=X^{\prime}$,

$$
\left|\left\langle K^{-1} a, K^{-1} b\right\rangle_{H_{1}}\right|=\left|\gamma\left(K^{-1} a, b\right)\right| \leq\left\|K^{-1} a\right\|_{\overline{X^{\star}}}\|b\|_{{\overline{X^{\star}}}^{\prime}}=\|a\|_{X^{\prime}}\|b\|_{{\overline{X^{\star}}}^{\prime}} .
$$

Therefore the theorem applies and we obtain $\left(X, \overline{X^{\star}}\right)_{\frac{1}{2}}=H_{1}$ with equality of norms.

\section{Applications}

In this section we show how the special cases mentioned in the introduction become easy corollaries of Theorem 1 .

Corollary 2. Let $H$ be a Hilbert space, let $v: X \rightarrow H$ be an injection with dense range, and let $Y=v(X)$ with norm $\|v(x)\|_{Y}=\|x\|_{X}$ for every $x \in X$. Let the duality between $Y$ and $Y^{\star}$ be given by a bilinear functional which extends the bilinear functional $\beta: Y \times \bar{H} \rightarrow \mathrm{C}, \beta(y, h)=\langle y, h\rangle_{H}$, so that $H \subset \overline{Y^{\star}}$. Then $\left(Y, \overline{Y^{\star}}\right)_{\frac{1}{2}}=H$ with equality of norms.

Proof. Here we have $Y \subset H \subset \overline{Y^{\star}}$ with $Y \cap \overline{Y^{\star}}=Y$ dense in $H$, $Y+\overline{Y^{\star}}=\overline{Y^{\star}}$, and $F$ is the closure of $Y$ in $\overline{Y^{\star}}$. Then $H \subset F$ densely so we can consider 


$$
\overline{F^{\star}}=\left\{y \in H,\left|\langle x, y\rangle_{H}\right| \leq C\|x\|_{F} \forall x \in H\right\} .
$$

We have

$$
\begin{aligned}
Y \subset \overline{F^{\star}} & \subset H \subset F \subset \overline{Y^{\star}}, \\
F^{\prime} & \subset H^{\prime} \subset \overline{F^{\star}} \subset Y^{\prime},
\end{aligned}
$$

and for every $a, b$ in $Y^{\prime} \cap F^{\prime}=F^{\prime}$ we have $K^{-1} a, K^{-1} b$ in $\overline{F^{\star}}$ and

$$
\left|\left\langle K^{-1} a, K^{-1} b\right\rangle_{H}\right| \leq\left\|K^{-1} a\right\|_{F}\|b\|_{F^{\prime}}=\left\|K^{-1} a\right\|_{\bar{Y}^{\star}}\|b\|_{F^{\prime}}=\|a\|_{Y^{\prime}}\|b\|_{F^{\prime}},
$$

hence the theorem applies and we get the result.

Corollary 3. Let $X$ be a Banach space compatible with $\overline{X^{\star}}$ such that $X \cap \overline{X^{\star}}$ is dense in $X$ and in $\overline{X^{\star}}$. Let $H$ be an intermediate Hilbert space between $X$ and $\overline{X^{\star}}$ with a scalar product compatible with the duality $\left(X, X^{\star}\right)$ and $X \cap \overline{X^{\star}}$ dense in $H$. If $K^{-1}$ maps $X^{\prime} \cap \overline{X^{\star}}$ into $\overline{X^{\star}} \cap X$ then $\left(X, \overline{X^{\star}}\right)_{\frac{1}{2}}=H$ with equality of norms.

Proof. Here we have $F=\overline{X^{\star}}$, and it is easy to check that $K$ maps $\overline{X^{\star}} \cap X$ into $X^{\prime} \cap \overline{X^{\star}}$ with $\|K x\|_{X^{\prime}}=\|x\|_{\overline{X^{\star}}}$ and $\|K x\|_{\overline{X^{\prime}}}=\|x\|_{X}$ for every $x \in X \cap \overline{X^{\star}}$. Let $a, b \in X^{\prime} \cap{\overline{X^{\star}}}^{\prime}$. Then by hypothesis $K^{-1} a, K^{-1} b \in X \cap \overline{X^{\star}}$ so that

$$
\left|\left\langle K^{-1} a, K^{-1} b\right\rangle_{H}\right| \leq\left\|K^{-1} a\right\|_{\bar{X}^{\star}}\left\|K^{-1} b\right\|_{X}=\|a\|_{X^{\prime}}\|b\|_{{\overline{X^{*}}}^{\prime}}
$$

and the theorem applies.

Before we state the next corollary let us explain the setting. A space $X$ is called a space of sequences if $X$ is a Banach space included in the space $\omega$ of all complex valued sequences such that the space $c_{00}$ of finitely supported sequences is dense in $X$ and the inclusion $X \rightarrow \omega$ is continuous with respect to the topology induced on $\omega$ by the family of semi-norms $p_{n}(x)=\left|x_{n}\right|$. We denote as usual by $e_{n}$ the sequence whose all coordinates are 0 except the $n^{\text {th }}$ which is equal to 1 . If $X$ is a Banach space with a basis $\left(b_{n}\right)_{n \geq 1}$, we identify $X$ with the space of sequences which is the completion of $c_{00}$ for the norm

$$
\left\|\sum_{k=1}^{n} x_{k} e_{k}\right\|=\left\|\sum_{k=1}^{n} x_{k} b_{k}\right\|_{X} .
$$

Then $i_{1}: \overline{X^{\star}} \rightarrow \omega$ which maps a functional $f$ to the sequence $\left(\overline{f\left(e_{n}\right)}\right)_{n \geq 1}$ is a continuous linear injection. We set $Y=i_{1}\left(\overline{X^{\star}}\right)$ and we norm $Y$ so that $i_{1}$ is an isometry. Then we decide to define the action of bounded linear functionals on $X$ in terms of 


$$
\begin{aligned}
\psi: X \times Y & \rightarrow \mathrm{C} \\
(x, y) & \mapsto \sum_{k=1}^{\infty} x_{k} \overline{y_{k}}
\end{aligned}
$$

so that we identify $\overline{X^{\star}}$ with $Y$. Now $X$ and $\overline{X^{\star}}$ are both subspaces of $\omega$ and $X \cap \overline{X^{\star}}$ is continuously embedded into $l^{2}$. If we assume moreover that $l^{2}$ is continuously embedded in $X+\overline{X^{\star}}$ then $l^{2}$ is intermediate between $X$ and $\overline{X^{\star}}$ and the scalar product of $l^{2}$ is clearly compatible with the duality $\left(X, X^{\star}\right)$. Also $X \cap \overline{X^{\star}}$ is automatically dense in $l^{2}$ and in $X$ since it contains $c_{00}$ which is dense in $l^{2}$ and in $X$. We consider as before the closure $F$ of $X \cap \overline{X^{\star}}$ in $\overline{X^{\star}}$, and we dualize the inclusions

$$
c_{00} \subset X \cap F \subset l^{2} \subset X+F \subset X+\overline{X^{\star}} \subset \omega
$$

into

$$
X^{\prime} \cap F^{\prime} \subset l^{\prime \prime} \subset X^{\prime}+F^{\prime},
$$

and we note that the duality $\gamma: X \cap F \times\left(X^{\prime}+F^{\prime}\right)$ is given by $\gamma(x, K h)=$ $\sum_{k=1}^{\infty} x_{k} \overline{h_{k}}$ for every $h \in l^{2}$, so that we can write

$$
\begin{aligned}
K: l^{2} & \rightarrow l^{2^{\prime}} \\
h & \mapsto \bar{h} .
\end{aligned}
$$

Now we are ready for Corollary 4 :

Corollary 4. Let $X$ be a Banach space with a basis $\left(b_{n}\right)_{n \geq 1}$ and let $\left(b_{n}^{\star}\right)_{n \geq 1}$ be the sequence of coefficient functionals. We assume that the projections

$$
\begin{gathered}
P_{N}: \overline{X^{\star}} \longrightarrow \overline{X^{\star}} \\
\sum_{k=1}^{+\infty} x_{k} b_{k} \star \longmapsto \sum_{k=1}^{N} x_{k} b_{k}^{\star}
\end{gathered}
$$

are of norm less than one for every $N$ and we interpolate $X$ and $\overline{X^{\star}}$ in the setting of sequence spaces as explained above. If in this setting we have moreover that $l^{2}$ is continuously embedded in $X+\overline{X^{\star}}$ then $\left(X, \overline{X^{\star}}\right)_{\frac{1}{2}}=l^{2}$ with equality of norms.

Proof. We only have to check the hypothesis of Theorem 1. Let $a$, $b \in X^{\prime} \cap F^{\prime}$. Then $a$ and $b$ are sequences and $\left\langle K^{-1} a, K^{-1} b\right\rangle_{l^{2}}=\sum_{k=1}^{\infty} \bar{a}_{k} b_{k}$. Now 


$$
\begin{aligned}
\left|\sum_{k=1}^{N} \bar{a}_{k} b_{k}\right| & =|\gamma(\bar{a}, b)| \leq\left\|P_{N}(\bar{a})\right\|_{F}\|b\|_{F^{\prime}}=\left\|P_{N}(\bar{a})\right\|_{\bar{X}^{*}}\|b\|_{F^{\prime}} \leq\|\bar{a}\|_{\overline{X^{*}}}\|b\|_{F^{\prime}} \\
& =\|a\|_{X^{\prime}}\|b\|_{F^{\prime}},
\end{aligned}
$$

and letting $N$ tend to $+\infty$ we obtain the desired inequality.

Before stating the last corollary let us recall (cf. [8]) that a Köthe function space on a complete $\sigma$-finite measure space $(\Omega, \Sigma, \mu)$ is a Banach space $X$ consisting of equivalence classes, modulo equality almost everywhere, of locally integrable functions such that:

1) if $g$ belongs to $X$ and if $f$ is a measurable function such that $|f(\omega)| \leq$ $|g(\omega)|$ a.e. on $\Omega$ then $f$ belongs to $X$ and $\|f\| \leq\|g\|$;

2) for every $\sigma \in \Sigma$ of finite measure the characteristic function $\chi_{\sigma}$ belongs to $X$.

Corollary 5. Let $X$ be a Köthe function space on a complete $\sigma$-finite measure space $(\Omega, \Sigma, \mu)$ such that $X \cap \overline{X^{\star}}$ is dense in $X, X$ and $\overline{X^{\star}}$ are intermediate between $L^{1}(\Omega)$ and $L^{\infty}(\Omega)$, and $L^{2}(\Omega)$ is intermediate between $X$ and $\overline{X^{\star}}$. Then $\left(X, \overline{X^{\star}}\right)_{\frac{1}{2}}=L^{2}(\Omega)$.

Proof. Here $X$ is a subspace of $L^{1}+L^{\infty}$, the map $i_{1}: \overline{X^{\star}} \rightarrow L^{1}+L^{\infty}$ is defined by $i_{1}(f)=\bar{f}$, and the map $\psi$ by $\psi(f, g)=\int_{\Omega} f \bar{g}$. The scalar product of $L^{2}$ is clearly compatible with the duality $\left(X, X^{\star}\right)$, and the map $K$ is given by $K(h)=\bar{h}$. Then we only have to check the inequality mentioned in Theorem 1. Let $a, b \in X^{\prime} \cap F^{\prime}$. Write $\Omega=\cup_{n=1}^{\infty} \Omega_{n}$ with $\mu\left(\Omega_{n}\right)<\infty$ for every $n$. Then $a_{n}=\chi_{\Omega_{n}} \chi_{\{|a| \leq n\}}$ is in $L^{1} \cap L^{\infty}$ hence in $X \cap \overline{X^{\star}} \subset F$ and $a_{n} \rightarrow a$ a.e. as $n$ tends to infinity with $\left|a_{n}\right| \leq|a|$. We have

$$
\left\langle K^{-1} a_{n}, K^{-1} b\right\rangle_{L^{2}}=\int_{\Omega} \overline{a_{n}} b d \mu
$$

and

$$
\left|\int_{\Omega} \overline{a_{n}} b d \mu\right| \leq\left\|\overline{a_{n}}\right\|_{F}\|b\|_{F^{\prime}}=\left\|\overline{a_{n}}\right\|_{\overline{X^{*}}}\|b\|_{F^{\prime}} \leq\|\bar{a}\|_{\bar{X}^{*}}\|b\|_{F^{\prime}}=\|a\|_{X^{\prime}}\|b\|_{F^{\prime}} .
$$

Now $\int_{\Omega} \overline{a_{n}} b d \mu \rightarrow \int_{\Omega} \bar{a} b d \mu=\left\langle K^{-1} a, K^{-1} b\right\rangle_{L^{2}}$ therefore $\left|\left\langle K^{-1} a, K^{-1} b\right\rangle_{L^{2}}\right| \leq$ $\|a\|_{X^{\prime}}\|b\|_{F^{\prime}}$ and the theorem applies.

ACKNOWLEDGEMENTS. I thank very much the referee for having pointed out the dangerous confusions contained in this paper before he made his careful report. 


\section{REFERENCES}

1. J. Bergh, On the relation between the two complex methods of interpolation, Indiana Univ. Math. J. 28 (1979), 775-777.

2. A. V. Bukhvalov, On the analytic Radon-Nikodym property, Proceedings of the second international conference, Poznan 1989, Teubner Text zur Math. 120, 1991, p. 211-228.

3. J. Bergh and J. Löfström, Interpolation spaces: an introduction, Springer-Verlag, 1976.

4. A. P. Calderón, Intermediate spaces and interpolation, the complex method, Studia Math. 24 (1964), 133-190.

5. F. Cobos and T. Schonbek, On a theorem by Lions and Peetre about interpolation between a Banach space and its dual, Houston J. Math. 24 (1998), 325-344.

6. U. Haagerup and G. Pisier, Factorization of analytic functions with values in noncommutative $L^{1}$-spaces and applications, Canad. J. Math. 41 (1989), 882-906.

7. S. G. Krein, Ju. I. Petunin and E. M. Semenov, Interpolation of linear operators, Nauka, Moscou, 1978; AMS Providence, 1981.

8. J. Lindenstrauss and L. Tzafriri, Classical Banach spaces II, Ergeb. Math. Grenzgeb. 97, 1979.

9. G. Pisier, The operator Hilbert space $\mathrm{OH}$, complex interpolation and tensor norms, Mem. Amer. Math. Soc. 122 (585), 1996.

10. H. Triebel, Interpolation theory, function spaces, differential operators, North-Holland Math. Library 18, 1978.

11. F. Watbled, Interpolation complexe d'un espace de Banach et de son antidual, C. R. Acad. Sci. Paris 321(I) (1995), 1437-1440.

12. F. Watbled, Ensembles de Rosenthal pour des fonctions à valeurs dans un espace de Banach. Interpolation complexe d'un espace de Banach et de son dual, Thèse de l'Université Paris 7, 1996.

13. T. Wolff, On the analytic Radon-Nikodym property, Harmonic Analysis, Lecture Notes in Math. 908 (1982), 199-204.

LMAM, UNIVERSITÉ BRETAGNE-SUD

1 , RUE DE LA LOI

56000 VANNES

FRANCE

Email : Frederique.Watbled@univ-ubs.fr 